Im Rahmen des «Forum Generica» vom 13. Januar 2005 in Zürich ist der FMH-Präsident eingeladen worden, einen Beitrag zum Thema des Forums «Vertragsfreiheit - eine vernünftige Strategie für die öffentliche Gesundheit in der Schweiz?» zu leisten. Hier sein für die Publikation leicht überarbeiteter Text.

\title{
«Vertragsfreiheit»: Steht dies noch zur Diskussion?
}

\author{
Die Zeit für Lösungen - das Engagement der Ärzteschaft
}

\section{Wer weiss also, was die Medizin ist?}

Man hat verschiedene Referenten zu diesem Forum, das der medizinischen Tätigkeit gewidmet ist, eingeladen: gesamthaft sieben Personen - darunter drei Ökonomen und ein Mediziner!

Man könnte manchmal meinen, die Medizin sei ein von ein paar Unternehmern, von «grossartigen» Visionären, auf den Markt gebrachtes Konsumgut, wie eine Leistung, die allein von den Wirtschaftswissenschaften ausreichend definiert wird.

Die Medizin ist jedoch keine rentable und automatisierte «Dienstleistung» und wird dies auch niemals sein, keine simple Leistung etwa, die man mit zwei Mausklicks berechnen könnte. Nein, es handelt sich hier viel eher um die bereits tausend Jahre währende Begleitung derjenigen, die körperlich oder seelisch leiden; es geht hier um das Leben, ein besseres Leben und die Kreativität eines jeden Einwohners, einer jeden Einwohnerin unseres Landes. Es handelt sich um eine Wissenschaft, eine Kunst, die sicherlich nicht die Welt, in der sie ausgeübt wird, ausser Acht lassen kann, aber die in keinem Fall Regeln unterworfen werden darf, die sie «entarten» und die sie wirkungslos machen, die aus ihr eine Hochstapelei, einen Schwindel betätigen - sie ist eine zarte und zerbrechliche Kunst, so dass der Ärztestand mittlerweile entschlossen ist, sie mit aller Kraft zu verteidigen, so sehr er von dem Wert seiner Tätigkeit überzeugt ist.

Man kann auch sagen, um es anders ... und polemischer darzustellen, was darüber hinaus nicht falsch ist, dass die Medizin sich offensichtlich an der Schnittstelle zwischen zwei Facetten der Gesellschaft befindet; an dem Berührungspunkt zwischen denjenigen, die einerseits die Gesell- schaft formen möchten, und andererseits denjenigen, die sie leben, sie ausmachen.

Genau an diesem Berührungspunkt schlagen die Funken: Es ist kein Zufall, dass die Medizin in der moderneren Welt Gegenstand absolut gegensätzlicher Forderungen zwischen Arbeitern und Arbeitgebern, zwischen Versicherern und Patienten, ist.

Sie werden leicht verstehen, wie wichtig in dieser Situation für uns eine totale Unabhängigkeit von allen Protagonisten des Gesundheitssystems ist - es geht hier nicht um das Ablehnen von Verantwortung, sondern es geht wirklich um die Unabhängigkeit: Wir können doch nicht einem Diktat, von wem auch immer, unterworfen werden, ohne zwangsläufig einen anderen Teil unserer Berufung zu verraten.

Was natürlich an der Stellung der Medizin in unserer Welt nichts bringt, die sich verzweifelt am Neo-Liberalismus versucht; die Medizin an sich ist eine weiche Wissenschaft, nennen Sie sie «soft» oder «fuzzy», wenn Sie mögen, eine Wissenschaft, wo das Wichtige häufig gefühlsbetont ist - das ist weder modern, noch konform mit dem vorherrschenden Ideologiemodell.

Das ist in der Tat nicht konform, das ist hochgradig suspekt, schreit nach Dilettantismus und öffnet jeder Art von Argwohn Tür und Tor, aber dies will etwas $z u$ den Prioritäten sagen, die die unseren sind; und an dieser Stelle wird es wieder interessant!

Es gibt selbstverständlich Prioritäten, die für die eine oder andere Ausrichtung der Medizin spezifisch sind, eher chirurgisch oder auf die Psyche bezogen, «sozial und präventiv», oder was weiss ich. Aber es gibt Prioritäten, die uns allen gemein sind und die so zentral sind, dass sie das Skelett, die Wirbelsäule sämtlicher medizinischer Fachgebiete sind. 
Das wäre nebenbei gesagt die Wiederentdeckung dieses gemeinsamen Kapitals, dieses «Familienkapitals», auf dessen Grundlage derzeit die FMH eine Einheit neu schmiedet. Und so ist es auch!

Diese Prioritäten sind derart grundlegend für die medizinische Praxis, dass sie für uns «obligatorisch» werden: So sehr wir mit Nachdruck ihre Berücksichtigung fordern, dass ihre Einhaltung seitens anderer Akteure des Gesundheitssystems gewährleistet wird, so sehr verpflichten sie auch uns selbst in allem, was die diesbezüglichen Anforderungen in der Ausübung unseres Berufes angeht.

Lassen Sie mich vier dieser Prioritäten anführen, und zwar beginnend - Sie werden nicht überrascht sein - mit

- der Tatsache, den zentralen Platz der zwischenmenschlichen Beziehung zwischen Arzt und Patient/Patientin beizubehalten; diese Beziehung ist eine grundlegende Aufgabe der Medizin und kein komischer Einfall der Postmoderne. Ich möchte Hippokrates zitieren um aufzuzeigen, dass bereits in jener Zeit die Beziehung zwischen Arzt und dem/der Kranken mehr sein konnte, als die simple Aufzählung von Fieber- oder Pustelproblemen. Sagte er nicht: «Gebe nicht das Geheimnis desjenigen weiter, der sich Dir anvertraut hat»? Wie kann besser aufgezeigt werden, dass bereits vor 2500 Jahren zu Beginn unserer Zivilisation die zwischenmenschliche Beziehung zur Medizin gehörte?

Diese starke und so einzigartige Beziehung, die zu unserem Beruf gehört, ist Teil der Therapie, sie muss absolut ihren Platz und ihren Wert behalten, und zwar im wahrsten Sinne des Wortes; sie muss ihren Raum behalten, den man als «Raum der relationalen Kreativität» bezeichnen könnte, denn nur dieser Raum macht eine gute Behandlung möglich; niemand ist berechtigt, ihn anzurühren, niemand hat das Recht, in ihn einzugreifen - vor allem nicht durch Beschränkung der freien Arztwahl...

- Des Weiteren ist innerhalb der Prioritäten, die als Motto voranzustellen sind, die Möglichkeit anzuführen, zu bewahren, was man unsere «therapeutische Kreativität» nennen könnte. Diese therapeutische Kreativität bedeutet, dass wir den Behandelnden wahrnehmen in einer subtilen Mischung, die der Intuition, der Kenntnisse und der Wahrnehmung des anderen in seiner Situation bedarf. Sie bedeutet, zu spüren was ihn heilen könnte, was seiner Situation entspricht, oder was, allen Leitlinien der Welt zum Trotz, nicht die präzise Wirklichkeit einer Situation darstellen wird.

Es gibt folglich, vor oder mit der therapeutischen Freiheit, die Freiheit zu denken, die Freiheit zu handeln, die Freiheit zu verordnen. Verstehen Sie bitte nicht falsch, damit ist nicht die Freiheit gemeint, alles zu tun, sehr wohl aber die Freiheit, die uns zur Verfügung stehenden Instrumente zu benutzen, um eine Situation zu entwirren und dann eine Lösung zu finden, die Freiheit, diese Instrumente mit gesundem Menschenverstand, aber auch kreativ zu verwenden.

Es ist anzumerken, dass laut den Erfahrungen der Ärzte, die Bedrohungen, die auf unserer beruflichen Zukunft lasten, unsere Kapazität, frei, d.h., ohne Zwang und Angst, zu denken, beeinträchtigen.

Und ich habe die Leitlinien angesprochen: Sie müssen genau dies sein, aber nicht mehr, eine Angabe, ein Leitfaden, eine Richtung, aber kein Kochrezept und noch viel weniger eine obligatorische Norm; man könnte hier ebenfalls die Gefahr anführen, mit der die Begrenzung des Leistungskataloges unsere therapeutische Kreativität bedroht, oder die Gefahr der Ärzte/Ärztinnen, a fortiori auf der Grundlage unangemessener, z. B. wirtschaftlicher Kriterien, ausgewählt worden zu sein.

- Eine andere, nicht minder wichtige Priorität ist es, die Qualität zu fördern und sicherzustellen.

Die Qualität der Leistungen einerseits - das Thema ist aber sehr viel umfangreicher: technische Leistungen wie das Labor oder die Radiologie, invasive Leistungen mit ihrem «outcome», Behandlung chronischer Patienten mit fortlaufender Kontrolle kritischer Parameter - Cholesterinspiegel oder Blutdruck im Laufe der Behandlung, der Prozentsatz der Besuche beim Augenarzt von Diabetikern etc., um nur einige Beispiele zu nennen.

Die Qualität der Leistungen einerseits, die Qualität des Praxisbetriebs andererseits, ein verheissungsvoller Bereich durch die Verbesserung der Behandlungen, die er verspricht, als auch durch die Einsparungen, die man über diesen Umweg anstreben kann, und schliesslich durch die Verbesserung der Lebensqualität der Ärzte/Ärztinnen. Es laufen vielzählige Projekte zu diesem Thema, davon das EPA-Projekt, an dem die FMH derzeit arbeitet.

Die Qualität der formellen oder informellen Netze, die die Ärzte/Ärztinnen aufbauen, ist ebenfalls ein Punkt, der zur Qualitätssteigerung des Praxisbetriebs gehört. - Ich verstehe 
unter informellen Netzen diejenigen, die ein jeder Arzt/jede Ärztin spontan um seine Praxis errichtet, das Netz der Kollegen/Kolleginnen, mit denen man regelmässig arbeitet und denen man vertrauen kann, Netze, die man zu häufig vergisst, wenn man sich an modernen Themen der Gesundheitspolitik ergötzt...

- Zu den für uns wichtigen Prioritäten, insbesondere zur Strukturierung unserer politischen Aktionen, - also unser Streben, dem Einfluss des «tout-économique», das uns erstickt, zu entkommen, - gehört die vierte und letzte Priorität. Diese Priorität ist für den Ärztestand, im sozialen Leben, das den Alltag der Patienten/Patientinnen ausmacht, verankert zu bleiben.

Eine durchaus wichtige Priorität, wenn es um die Strukturierung unserer politischen Aktionen geht. Denn eine schreckliche Bedrohung der Ärzte ist es seit Jahrhunderten, an Gesellschaftsmodellen festzuhalten, die bereits für jeden, nur nicht für die Ärzte und Ärztinnen, überholt sind, und sich so von Dialog- und Handlungsmöglichkeiten zu beschneiden, die positiver als der defensive Rückzug sind. Zur Erläuterung nur ein Beispiel: In dem sozialen Leben unserer Zeit verankert zu sein, bedeutet in einer Zeit, wo niemand mehr ein Schokoladenbrötchen oder eine Konservendose kauft, wenn dort nicht sämtliche Zutaten und ein Verfallsdatum aufgeführt sind, dass man einfach nicht gewillt sein kann, medizinisch tätig zu sein, ohne dass unsere «Kunden und Kundinnen» von der Qualität unserer Leistungen und Ausbildung überzeugt sind - das ist völlig normal und hier müssen wir ansetzen, anstatt weiterhin einer nicht mehr vertretbaren Illusion zu verfallen, dass die alleinige Tatsache, dass man «Doktor» ist, man somit über jegliche Kontrolle erhaben ist.

Die Information und das «empowerment» der Patienten und Patientinnen gehören zu demselben Register, dem Register der Realitäten und Gegebenheiten, mit denen man leben kann und muss, wie auch die Wirtschaftlichkeit gegenüber den Institutionen - den Versicherern, und vor allem, dem Staat - die den wesentlichen Anteil der von uns ausgestellten Rechnungen tragen.

Bitte beachten Sie, dass ein Leben in und unter Berücksichtigung einer aktuellen Realität nicht heissen soll, alles stumm hinzunehmen, es kann jedoch nicht oder nicht mehr angehen, hochmütig abzulehnen, zur Sache zu kommen.
Kurzum - in der sozialen Realität verankert zu bleiben, oder vielmehr - leider - sich dort neu verankern zu müssen, ist eine Voraussetzung für zwei Dialogformen, denen wir besonders verpflichtet sind, und zwar auf zwei unterschiedlichen Ebenen: der Dialog mit unseren Partnern des Gesundheitssystems und, natürlich, der Dialog mit den Patienten/Patientinnen.

\section{Die FMH und die KVG-Revision}

Die FMH hat sich auf diese Überzeugungen stützend einen für sie neuen Weg gewählt, nämlich profilierte öffentliche Ansichten in Kauf zu nehmen und folglich ihre aktive Präsenz in der Politik derart zu behaupten, dass keine Gesundheitspolitik mehr in unserem Land ohne die FMH gemacht wird.

Wir haben dies derart beurteilt, dass im Rahmen der Debatte über die vom KVG - eingeschnürte - Revision konkrete Vorschläge zu machen sind. Denn die politische Welt, die sich zwar auf reale Probleme stützt, aber auch auf Interpretationen die bestenfalls diskutierbar und schlimmstenfalls eindeutig inakzeptabel sind, diese politische Welt ist so weit gekommen, Lösungen vorzubringen, über die es für uns leider nicht in Frage kommt zur Sache zu kommen.

Konkret bedeutet dies, dass in der Diskussion über die freie Arztwahl die realen Probleme darin bestehen, die Qualität der Ausbildung der Ärzte und der erbrachten Leistungen sowie die diesbezügliche Aufklärung der Patienten/Patientinnen zu garantieren und die Wirtschaftlichkeit der medizinischen Tätigkeit zu fördern.

Abgesehen davon geht es hier nicht einfach darum, um diese beiden tatsächlichen Probleme zu lösen, die freie Arztwahl über Bord zu werfen und bei dieser Gelegenheit auf eine Absicherung des Arbeitsplatzes und der Existenz all unserer Kollegen und Kolleginnen, die gut arbeiten, zu verzichten. - Und die, die gut arbeiten, und dies ist ja unbestritten, sind die Quasitotalität des Ärztestandes. Wir werden niemals eine Entscheidung über Leben oder Tod unserer Karriere in den Händen von Verwaltungen belassen, die nicht so oft, weder für die Qualität ihrer Arbeit, noch für die Korrektur, die Kohärenz ihrer Haltungen berühmt sind.

Wir schlagen deshalb die Beibehaltung des automatischen Ablaufs der Zulassung der Ärzte zur Erstattung ihrer Rechnungen vor, denn so wird der Ärztestand vor der Willkür einer Krankenkasse oder einer anderen geschützt. Für die Lösung der oben angeführten Probleme schlagen wir hinge- 
gen vor, dass diese Zulassung zur Erstattung der Rechnungen durch die Sozialversicherung $z u$ sätzlichen Kriterien unterworfen werden kann, die über die aktuellen Basiskriterien (das Arztdiplom und zwei Jahre Praktikum) hinausgehen:

Der Bundesrat kann die Zulassung von Ärzten und Ärztinnen von der Erfüllung zusätzlicher Kriterien bezüglich Qualität und Wirtschaftlichkeit abhängig machen.

Selbstverständlich ist es unbedingt erforderlich, wenn die Zeit kommt, dass die Ärzte - die Einzigen, die wissen, was Medizin bedeutet! - diese Kriterien selbst ausarbeiten; niemand anders darf uns diese Kriterien auferlegen! Damit jedoch bei dieser Vorgehensweise die Glaubwürdigkeit gegeben ist und keine einseitige berufsständische Interessenvertretung vorgeworfen werden kann, schlagen wir vor, dass diese Kriterien nicht alleinig von den Ärzten ausgearbeitet werden, sondern gemeinsam mit den Versicherern. - Das Ergebnis einer derartigen Zusammenarbeit kann nur adäquat und effizient sein:

Der Bundesrat beauftragt die Tarifpartner mit der Ausarbeitung dieser gesamtschweizerisch einheitlichen Kriterien und genehmigt diese.

Können die Ärzte und Versicherer letztendlich keine Einigung erzielen, schlagen wir vor, dass eine eidgenössische Ad-hoc-Kommission die Kriterien ausarbeitet, eine Kommission bestehend aus Vertretern der Ärzte und Versicherer, aber auch zwecks Erreichung eines positiven und anerkannten Ergebnisses, aus Vertretern und Vertreterinnen der Kantone und der Patienten und Patientinnen:

Können sich die Tarifpartner nicht einigen, so legt der Bundesrat diese Kriterien fest. Er stützt sich dabei auf die Vorschläge einer paritätischen Kommission.
Die FMH ist der Meinung, eine Lösung zu präsentieren, die effizient und glaubwürdig auf die Besorgnisse eingeht, die sich derzeit hinter der Besessenheit unserer Politiker und Politikerinnen und Versicherer zur so genannten «Vertragsfreiheit» verbergen, eine Lösung zur Aufrechterhaltung einer automatischen Zulassung zur Rückerstattung seitens der Sozialversicherung, für uns eine sine qua non Bedingung.

Wir können unsere verschiedenen Partner nicht eindringlich genug bitten, die Wichtigkeit dieses Projekts der FMH zu erkennen. Es stellt eine wahre Lösung dar und ist im Vergleich zur Vergangenheit ein immenser Schritt nach vorne für den Ärztestand. Ein Schritt nach vorne, dessen Nichtbeachtung sehr bedauerlich wäre. Auf diese Art und Weise könnte ein Referendum vermieden werden. Ein Referendum, dass wir mit grosser Wahrscheinlichkeit gewinnen würden, was aber niemandem etwas bringen würde, ... und uns alle nur zum Anfang zurückwerfen würde!

Um abschliessend auf den Titel dieser Ausführungen zurückzukommen: "Aufhebung des Vertragszwanges: steht dies noch zur Diskussion?», für uns ist die Antwort nein!

Und zwar ein energisches, bestimmtes Nein, solide untermauert von gut begründeten Überzeugungen und darüber hinaus ein konstruktives Nein.

Der Untertitel lautete: «Die Zeit für Lösungen - das Engagement der Ärzteschaft». Sie haben die Vorschläge der FMH gehört; die Ärzte und Ärztinnen engagieren sich auf bemerkenswerte und neue Weise in der Politik. Sie sind bereit, sich eine Zukunft der Öffnung und Kreativität zu bauen, eine Zukunft, in der sie weiterhin einen Beruf ausüben, an den sie mit Vertrauen und Enthusiasmus glauben wollen. 